\title{
HOTAIR plays an oncogenic role in gastric cancer through microRNA and SNP
}

\author{
Minireview
}

Hui-Wen XU, Yi-Ru CHEN, Su-Shan OUYANG, Ping LI, Mei-Qian WANG, Sen-Lin ZHU*

Department of Gastroenterology and Hepatology, The First Affiliated Hospital, Sun Yat-sen University, Guangzhou, China

${ }^{*}$ Correspondence: zhusl@mail.sysu.edu.cn

Received January 27, 2021 / Accepted March 13, 2021

\begin{abstract}
HOX transcript antisense intergenic RNA (HOTAIR) is a lncRNA with a length of 2,158 nucleotides and its two terminal domains could combine with different complexes to function at the level of transcription and translation. It overexpresses in many cancers including gastric cancer. HOTAIR could play an oncogenic role in the initiation and progression of gastric cancer through interaction with microRNAs, such as miR-330/618/126 in the PI3K/Akt signaling pathways. HOTAIR single nucleotide polymorphisms (SNPs) may have genotype-function and allele-specific effect on gastric cancer by a mechanism that specific SNP could give rise to a variation of HOTAIR and alter the binding site of microRNAs. Both rs920778 T allele and rs4759314 G allele will enhance the susceptibility to gastric cancer in the Chinese populations. In a word, the suppression of HOTAIR and overexpression of downstream microRNAs may be potential therapeutic strategies of gastric cancer related to HOTAIR.
\end{abstract}

Key words: HOTAIR, gastric cancer, microRNA, associated SNPs

HOTAIR is an oncogenic lncRNA. Long non-coding RNAs (lncRNAs) are classified into many subgroups on the basis of their orientation and location, such as sense/ antisense, divergent/convergent, and intronic/intergenic types [1]. HOX transcript antisense intergenic RNA (HOTAIR), a lncRNA with a length of 2,158 nucleotides and limited protein-coding potential, is localized in the 5' HOXC locus on chromosome 12q13.13 and only one strand antisense to HOXC genes is transcribed, which is the first introduction to HOTAIR [2]. Two terminal domains of HOTAIR could combine with different complexes to function at the level of transcription and translation. The 5' domain of HOTAIR could directly bind to the enhancer of zeste homolog 2 (EZH2), the catalytic subunit of Polycomb Repressive Complex 2 (PRC2), while the 3' domain of HOTAIR could directly bind to lysine specific demethylase 1 (LSD1) belonging to the histone demethylase complex by observing the distribution of map of HOTAIR deletion mutants, suggesting that HOTAIR plays a role as a modular scaffold in different complexes. Then the two combinations resulted in histone H3 lysine 27 (H3K27) tri-methylation and histone $\mathrm{H} 3$ lysine 4 (H3K4) demethylation, the respective histone methylation products of PRC2 and LSD1 complexes, thereby maintaining a transcriptional inactivation in trans over the HOXD locus on chromosome 2 at last $[3,4]$.

The relative expression level of HOTAIR in cancer-associated samples could be quantified by quantitative real-time polymerase chain reaction (qRT-PCR) and $2-\Delta \Delta$ Cq method [5]. The first HOTAIR overexpression was found between primary breast cancer and metastases compared with normal human breast epithelia by qRT-PCR [6]. Besides, HOTAIR bound to PRC2 and altered the occupancy pattern and chromatin state, which could promote breast cancer metastasis to the lung [6]. A lot of reviews recapitulated that the overexpression of HOTAIR could also be found in many other cancers including colorectal cancer, hepatocellular cancer, gastrointestinal stromal tumor, laryngeal squamous cell carcinoma, lung cancer, pancreatic cancer, and gastric cancer $[4,7]$. These findings could elucidate that HOTAIR is an oncogenic lncRNA. However, the overall biological role and clinical significance of HOTAIR in gastric carcino- 
genesis remain largely unknown, we therefore focused on its role in gastric cancer.

HOTAIR promotes the initiation and progression of gastric cancer. Gastric cancer ranked the fourth in the incidence rate and the third in mortality rate among all malignant tumors worldwide $[8,9]$. The expression level of HOTAIR in the gastric cancer samples was diverse. Highly different expression levels of HOTAIR between 31 paired gastric adenocarcinoma tissues and normal adjacent gastric epithelium tissues were found and HOTAIR expression level had positive correlations with the expression level of suppressor of zeste 12 (SUZ12), TNM stages, and lymph node metastasis [10]. The concentration level of three lncRNAs H19, HOTAIR, and metastasis associated lung adenocarcinoma transcript-1 (MALAT1) were all detected to be low and stable in the 43 gastric cancer plasma samples, however, only plasma H19 levels were significantly higher compared with controls [11]. In contrast, in a recent study, the plasma levels of HOTAIR were significantly higher in 50 gastric cancer patients compared with corresponding healthy controls, and the plasma levels of HOTAIR as a diagnosis method had $88 \%$ sensitivity and $84 \%$ specificity [12].

HOTAIR was overexpressed in the gastric cancerous tissues and was associated with invasion and apoptosis abilities [13]. In addition, silencing HOTAIR could inhibit cell proliferation and motility, reverse epithelial-mesenchymal transition (EMT), arrest the G0/G1 of the cell cycle but induce apoptosis in gastric cancer, demonstrating that HOTAIR could be an indicator of carcinogenesis [14]. In another study, the total expression level of HOTAIR was higher but that of miR-454-3p was lower in gastric cancer tissues than non-tumor tissues. Knockdown of HOTAIR increased miR-454-3p to suppress gastric cancer proliferation and promote cell apoptosis by targeting STAT3/Cyclin D1 [15]. Therefore, HOTAIR associated with multiple oncogenic properties could play an oncogenic role in gastric cancer and would be a potential biomarker for it.

The oncogenic properties, like EMT and invasion, contribute to gastric carcinogenesis and metastasis. The expression levels of HOTAIR in 56 out of 83 paired human gastric cancer tissues were significantly higher than corresponding adjacent non-cancerous tissues and the morphological changes of EMT in cancer tissues were found [16]. After transfected with siRNA of HOTAIR, morphological changes similar to epithelial cells were revealed in AGS cells, which further suggested that HOTAIR was implicated in the initiation and progression of gastric cancer [16]. HOTAIR induced cancer cell migration and invasion through a combination with EZH2 to E-cadherin promoter directly to repress E-cadherin via RNA-binding protein immunoprecipitation and chromatin immunoprecipitation assay [17]. Noteworthy, in the latest study, using immunofluorescence staining to find E-cadherin-related morphological changes, Song et al. found that HOTAIR promoted EMT in gastric cancer through the epigenetic regulation of gene expression, such as through an increased switch of $\mathrm{H} 3 \mathrm{~K} 27$ acetylation to methylation at the E-cadherin promoter in the dependence of SUZ12, another subunit of PRC2 [18]. Thus, HOTAIR promotes the initiation and progression of gastric cancer through an increased change of EMT and enhanced invasive ability.

It is a noticeable phenomenon that HOTAIR is correlated with the specific histological type of cancer. For instance, HOTAIR expression levels were higher in the diffuse-type gastric cancer relative to the levels in the intestinal type, and subjects with high HOTAIR expression group had a significantly worse prognosis than those with low expression group in diffuse-type gastric cancer but not in intestinal-type [19]. Besides, the poor prognostic indicator of overexpressed HOTAIR in breast cancer patients was restricted to estrogen receptor (ER)-negative and node-positive patients [20]. It is worth further exploring the role of HOTAIR in specific histological types of cancer in the future.

HOTAIR results in carcinogenesis and cisplatin resistance by sponging microRNA. There were emerging numbers of studies suggesting that the interaction of HOTAIR with microRNAs could change the expression levels of their target genes and state of signaling pathways to promote initiation and progression of gastric cancer.

HOTAIR was identified to upregulate the expression of human epithelial growth factor receptor 2 (HER2) protein by sponging miR-331-3p in a competitive endogenously manner [13]. HOTAIR directly combined with EZH2 made a miR-34a silence epigenetically, resulting in C-Met and Snail activation successively that could repress $\mathrm{CDH} 1$ (also as E-cadherin) transcription to promote EMT in gastric cancer [19]. HOTAIR promoted invasion and gastric cancer growth by sponging miR-1277-5p and upregulating collagen type $\mathrm{V}$ al chain (COL5A1), providing novel targets for gastric cancer treatment [21]. The inhibitory effect of HOTAIR on miR-30a/b could enhance the expression of target genes KRAS and VEGF-A, which promote the invasion and angiogenesis of gastric cancer cells [22]. Besides, HOTAIR accelerated the biological activities of gastric cancer cells by regulating miR-206 - Cyclin D1(CCND1)/ Cyclin D2 (CCND2) signal pathway axis [23].

Moreover, miR-34a is not only closely associated with EMT but also with cisplatin resistance. Cheng et al. found that HOTAIR was over-expressed and miR-34a was low-expressed in 27 pairs of gastric cancer tissues compared with adjacent normal tissues and that HOTAIR directly bound to miR-34a by Luciferase reporter assay and RNA immunoprecipitation assay [24]. This combination could result in cisplatin resistance, change apoptosis-related genes and multidrug-resistance genes, and modify the phosphoinositide 3-kinases (PI3K)/protein kinase B (PKB, also as Akt) and Wnt/ $\beta$-catenin signaling pathways [24]. Similarly, HOTAIR promoted cisplatin resistance by directly binding to miR-126 to activate the PI3K/Akt/ multidrug resistance-associated protein 1 (MRP1) axis, suggesting HOTAIR was closely related with cisplatin resistance [25]. 
The PI3K/Akt signaling pathway was vital in the onset and procession of gastric cancer. Focusing on the interactions among HOTAIR, miR-618, and Krüppel-like factor 12 (KLF12) how affect cell viability, migration and invasion, cell apoptosis, mice xenograft models, and the ratios of $\mathrm{p}-\mathrm{PI} 3 \mathrm{~K} /$ PI3K and p-Akt /Akt, Xun et al. found upgraded HOTAIR and KLF12 but degraded miR-618 in gastric cancer tissues. HOTAIR promoted gastric cancer progression through the PI3K/ Akt signaling pathway via miR-618/KLF12 axis [26]. Similarly, the combination between HOTAIR and miRNA330 upregulated expression of Erb-B2 Receptor Tyrosine Kinase 4 (ERBB4, also as HER4) which could increase phosphorylation of the downstream PI3K/Akt pathway protein, resulting in resistance of gastric cancer cells to trastuzumab [27].

Not only does HOTAIR target specific microRNA, but also specific microRNA targets HOTAIR. For example, miR-101-3p functioned as a tumor suppressor to suppress proliferation and invasion in gastric carcinoma cells by directly targeting the serum response factor (SRF) gene and suppressing the function of SRF as a transcription factor of HOTAIR [28]. There will be a potential network between HOTAIR with microRNAs through signaling pathways to clarify the role of HOTAIR in gastric cancer onset and procession (Figure 1) so that we would develop novel clinic treatment against gastric cancer associated with HOTAIR.
HOTAIR SNPs have diverse effects on gastric cancer. Single nucleotide polymorphism (SNP) mainly refers to the DNA sequence polymorphism caused by single nucleotide variation at the genomic level, which is a heritable variation usually involving a single base conversion. SNP within HOTAIR can regulate HOTAIR gene expression and function at the level of genetic variations due to the intron or exon location of SNP, modulating individual susceptibility to various cancers [29].

HOTAIR was located on the opposite strand within the intergenic region between HOXC11 and HOXC12 in the HOXC gene cluster on chromosome 12q13.13 [2]. HOTAIR with a length of 2,158 nucleotides had several common SNPs. We pay attention to the association between HOTAIR SNPs and gastric cancer and find dissimilar risk caused by the same SNP in various ethnicities, hence we gather this association based on countries in this review (Table 1).

By collecting two independent hospital-based casecontrol sets in China (one set was discovery set consisting of 500 gastric cancer patients and 1,000 healthy controls, another was validation set consisting of 300 patients and 1,000 controls), Pan et al. found only rs920778 SNP located in a HOTAIR enhancer of intron 2 was significantly associated with the susceptibility of gastric cancer among rs920778, rs1899663, and rs4759314 SNPs. Besides, rs920778 TT genotype carriers had strikingly higher HOTAIR expres-

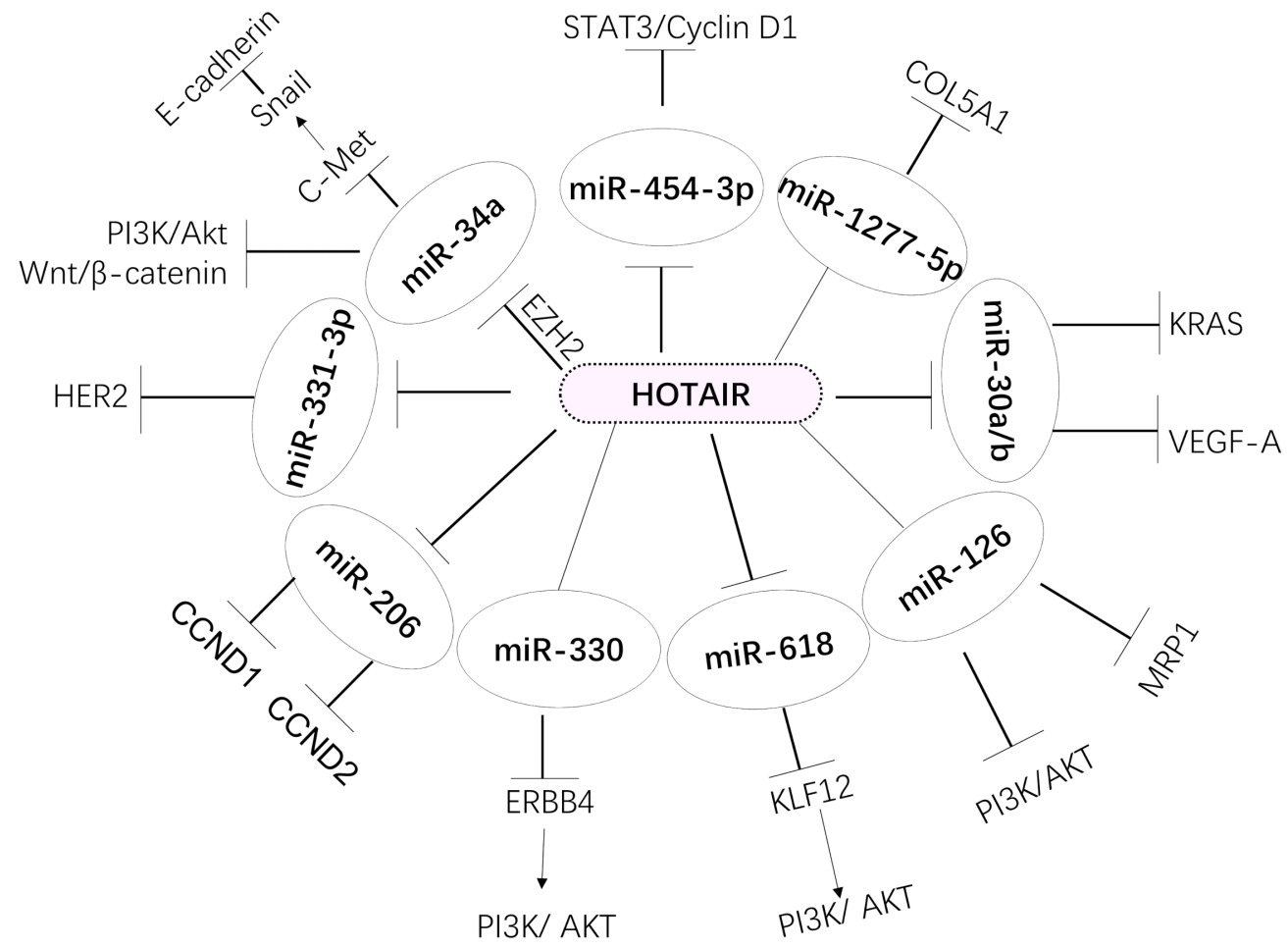

Figure 1. HOTAIR plays an oncogenic role in gastric cancer by sponging various microRNAs. The interaction between HOTAIR and miRNAs could result in the over-expression of miRNA targets and subsequent pathogenic events in gastric cancer. 
Table 1. HOTAIR SNPs have diverse effects on gastric cancer.

\begin{tabular}{|c|c|c|c|c|c|}
\hline Country & Sample size & Susceptibility to GC & Genotype or allele & Location & Reference \\
\hline Jinan, China & $\begin{array}{l}500 \mathrm{GC} ; \\
1000 \mathrm{HC}\end{array}$ & rs920778 & TT than CC or CT & enhancer of intron 2 & {$[30]$} \\
\hline Jiangsu, China & $\begin{array}{l}753 \mathrm{GC} \\
1057 \mathrm{HC}\end{array}$ & rs4759314 & AG than AA & HOTAIR intron 1 & {$[31]$} \\
\hline Hebei, China & $\begin{array}{l}515 \mathrm{GCA} \\
654 \mathrm{HC}\end{array}$ & rs12826786 & $\mathrm{TT}$ than $\mathrm{CC}$ or $\mathrm{CT}$ & HOTAIR promoter & {$[32]$} \\
\hline Elazig, Turkey & $\begin{array}{l}105 \mathrm{GC} \\
207 \mathrm{HC}\end{array}$ & rs12826786 (ns) & T allele & - & {$[34]$} \\
\hline Elazig, Turkey & $\begin{array}{l}104 \mathrm{GC} \\
209 \mathrm{HC}\end{array}$ & rs920778 (ns) & C allele & - & {$[35]$} \\
\hline Tokyo, Japan & $164 \mathrm{GC}$ & rs920778 (ns) & C allele & - & {$[36]$} \\
\hline Zhejiang, China & $\begin{array}{l}245 \mathrm{GC} \\
245 \mathrm{HC}\end{array}$ & rs17720428 & G allele & 3' UTR of HOTAIR & {$[37]$} \\
\hline Ardabil, Iran & $\begin{array}{l}300 \mathrm{GC} \\
300 \mathrm{HC}\end{array}$ & $\begin{array}{c}\text { rs17720428 } \\
\text { rs7958904 } \\
\text { rs1899663 }\end{array}$ & $\begin{array}{l}\text { G allele } \\
\mathrm{C} \text { allele } \\
\mathrm{T} \text { allele }\end{array}$ & $\begin{array}{l}- \\
- \\
-\end{array}$ & {$[38]$} \\
\hline $\begin{array}{l}\text { Europe (Lithuania, } \\
\text { Latvia, Germany) }\end{array}$ & $\begin{array}{l}613 \mathrm{GC} ; \\
118 \mathrm{AG} ; \\
476 \mathrm{HC}\end{array}$ & rs17840857 (ns) & G allele & - & {$[39]$} \\
\hline
\end{tabular}

Abbreviations: GC - gastric cancer; HC - healthy control; GCA - gastric cardia adenocarcinoma; AG - atrophic gastritis; ns - not significant

sion level than CC genotype or CT genotype in normal tissues [30]. In addition to this, only rs4759314 SNP located in HOTAIR intron 1 had a significant association with gastric cancer susceptibility (especially in the elder and male subjects) among rs4759314 A>G, rs7958904 G>C, rs874945 $\mathrm{G}>\mathrm{A}$ in a Chinese study including 753 cancer cases and 1,057 matched controls for test set [31]. Subjects with rs4759314 AG genotype had higher HOTAIR and HOXC11 expressions level than those with AA genotype in 63 gastric cancer tissues, so the $G$ allele could enhance promoter activity of HOXC11 in two cancer cell lines, which elucidated the genotype-function and allele-specific effect of HOTAIR rs4759314 [31]. Located in the promoter region of HOTAIR, rs12826786 C $>\mathrm{T}$ polymorphism had a functional effect on HOTAIR expression. The frequency of the $\mathrm{T}$ allele was significantly higher in 102 gastric cardia adenocarcinoma tissues than corresponding normal tissues in a retrospective casecontrol study in north China. When compared with subjects with CC and CT genotypes, subjects with the rs12826786 TT genotype showed a significantly higher expression level of HOTAIR, which indicated that $\mathrm{C}$ to $\mathrm{T}$ transition may influence the transcription and expression of the HOTAIR gene [32]. Consequently, the genotype-function and allele-specific effect of specific HOTAIR SNP, like rs920778 TT, rs4759314 AG, rs12826786 TT, deserve great value in China according to the result of the three Chinese large sample studies.

In addition to Chinese researches, other countries have focused on the association between the same HOTAIR SNPs and cancer. HOTAIR rs12826786 $\mathrm{C}>\mathrm{T}$ polymorphism in both codominant and recessive inheritance models had a significant association with a higher susceptibility to breast cancer and the TT genotype had an association with multiple clinic-pathological characteristics in the Turkish population
[33]. However, HOTAIR rs12826786 C>T polymorphism didn't played any major role in genetic vulnerability to gastric carcinogenesis within the Turkish population [34]. Conspicuously, in a hospital-based case-control study from Turkey consisting of 104 gastric cancer subjects and 209 healthy control subjects matched on age and gender, no significant association between HOTAIR rs920778 three genotypes and the risk of gastric cancer susceptibility and even by subgroups analysis was found [35], which was inconsistent with the role of rs920778 SNP in Chinese subjects [30]. By consecutively collecting 827 cancer positive and 546 cancer negative autopsy cases from a Japanese geriatric hospital, rs920778 didn't contribute to overall cancer susceptibility except for lung cancer type; the reason why this result wasn't consistent with other studies may be identified by a minor allele in a different way and in different ethnic populations [36].

Recently, other single nucleotide polymorphisms associated with gastric cancer were detected. Located in 3' UTR (untranslated region) of HOTAIR gene, rs17720428 had a significantly high risk for developing gastric cancer in Chinese subjects and especially in males, and subjects with rs17720428 minor $\mathrm{G}$ allele had higher expression levels of plasma HOTIAR and hsa-mir-5004-3p and lower expression level of KLF4 protein, which was accompanied with poor overall survival [37]. HOTAIR rs17720428, rs7958904, and rs1899663 SNPs and their interactions with the HOTTIP rs1859168 were significantly associated with gastric cancer risk in Ardabil (a very high-risk area of gastric cancer in north-western Iran) [38]. Thus, the similar manifestation between rs17720428 minor G allele and rs4759314 G allele [31] may have a synergistic effect on gastric cancer. It was first reported that the HOTAIR rs $17840857 \mathrm{G}$ allele had higher (but not statistically) susceptibility to atrophic 
gastritis and gastric cancer compared with the T allele in 118 atrophic gastritis patients and 613 gastric cancer patients from Europe [39].

These common types of HOTAIR single nucleotide polymorphisms were positively related or not related to the specific cancer type and a different population based on the above results. Therefore, we focused on the meta-analysis on SNPs to find conclusive result as much as possible.

Although no significant association between the HOTAIR rs4759314 polymorphism and cancer risk was found in the whole meta-analysis consisting of five articles of the Chinese population (including seven case-control studies), rs4759314 $\mathrm{G}$ allelic gene may augment the genetic risk for gastric cancer [40]. Besides, we pay attention to more analyses on types of SNPs. To find the association between HOTAIR rs920778 $\mathrm{C}>\mathrm{T}$, rs4759314 A>G, rs7958904 G>C, and rs1899663 $\mathrm{G}>\mathrm{T}$ polymorphisms and cancer susceptibility, Zhang et al. conducted a meta-analysis consisting of eight eligible articles in the Chinese and Turkish population and found significant associations between the HOTAIR rs920778 and cancer risk in eight Chinese studies [41]. HOTAIR rs4759314 only increased risk for the gastric cancer subgroup in eight Chinese studies. However, HOTAIR rs7958904 CC genotype significantly decreased susceptibility to cancer and colorectal cancer subgroup in three Chinese studies. HOTAIR rs1899663 G>T polymorphism didn't have any association with cancer risk in the meta-analysis [41]. Similarly, in a meta-analysis, HOTAIR rs920778 and rs874945 significantly increased and rs7958904 decreased cancer risk. Moreover, rs920778 was significantly susceptible to both the Chinese population and gastric cancer [42].

Analogical results of another meta-analysis were discovered. Making a meta-analysis from ten eligible studies consisting of seven kinds of HOTAIR single nucleotide polymorphisms (rs4759314, rs920778, rs7958904, rs1899663, rs874945, rs12826786, rs10783618) and Chinese/Turkish population, Qi et al. found that rs920778 significantly associated with cancer susceptibility in the Chinese population via stratified analysis based on ethnicity [43]. HOTAIR rs4759314 had a significant association with only gastric cancer type in four types of cancers in the meta-analysis. HOTAIR rs 920778 $\mathrm{T}$ allele increased risk for all studied cancers and rs4759314 $\mathrm{G}$ allele enhanced susceptibility to gastric cancer by allele contrast [43]. Based on the latest meta-analysis from nine publications about five HOTAIR polymorphisms (rs920778, rs7958904, rs874945, rs1899663, and rs4759314) with cancer susceptibility, it can draw the same conclusion as the above result: rs920778 polymorphism increased but rs7958904 polymorphism decreased the risk to cancer in the Chinese population, while none of the other three polymorphisms was significantly associated with cancer susceptibility [44].

According to the comprehensive result of the five metaanalyses, it was conclusive that rs920778 T allele and rs4759314 G allele could boost the susceptibility to gastric cancer in the Chinese populations and that rs7958904 CC genotype will play the protective role in gastric cancer in other populations. Both rs1899663 and rs874945 have no association with gastric cancer in the Chinese and Turkish populations. HOTAIR rs12826786 and rs10783618 remain to be further studied.

The limitations of publications about single nucleotide polymorphisms with cancer susceptibility were concluded. First, most studies were retrospective case-control studies in an ethnically homogeneous population, so selection bias might affect the conclusions of the related investigation. Second, because the number of samples in the stratification analysis was a small size, it is easy not to have enough power in terms of statistics. Third, the reported studies generally focused on single genetic variation but ignored gene-environment and gene-gene interactions. Finally, more prospective studies with a large sample size are needed to be carried out in the future that concentrate on the genotypefunction and allele-specific effect of specific SNP on specific cancer in different population-based studies.

Conclusion and future perspectives. In summary, HOTAIR promotes the initiation and progression and results in cisplatin resistance of gastric cancer through microRNAs and signaling pathways, such as miR-330/miR-618/miR-126 in the PI3K/Akt signaling pathways, meaning that it could be used as a potential biomarker and therapeutic target. To our knowledge, this is the first review to recapitulate the association between different HOTAIR SNPs and gastric cancer. HOTAIR SNPs may exert genotype-function and allele-specific effect on cancer in a manner that specific single nucleotide polymorphism might cause a variation of HOTAIR, and consequently change the binding site of microRNAs and modify microRNAs in different signaling pathways. Both rs920778 T allele and rs4759314 G allele will enhance the susceptibility to gastric cancer in the Chinese populations. A lot of prospective studies with a large sample size are needed to be carried out among different ethnic populations in the future.

Acknowledgments: This review was supported by Guangzhou Science and Technology Development Funds (Key Program, No. 201803010103).

\section{References}

[1] RINN JL ,CHANG HY. Genome regulation by long noncoding RNAs. Annu Rev Biochem 2012; 81: 145-166. https:// doi.org/10.1146/annurev-biochem-051410-092902

[2] RINN JL, KERTESZ M, WANG JK, SQUAZZO SL, XU X et al. Functional demarcation of active and silent chromatin domains in human HOX loci by noncoding RNAs. Cell 2007; 129: 1311-1323. https://doi.org/10.1016/j.cell.2007.05.022

[3] TSAI MC, MANOR O, WAN Y, MOSAMMAPARAST N, WANG JK et al. Long noncoding RNA as modular scaffold of histone modification complexes. Science 2010; 329: 689-693. https://doi.org/10.1126/science.1192002 
[4] CAI B, SONG XQ, CAI JP, ZHANG S. HOTAIR: a cancer-related long non-coding RNA. Neoplasma 2014; 61: 379-391. https://doi.org/10.4149/neo_2014_075

[5] LIVAK KJ, SCHMITTGEN TD. Analysis of Relative Gene Expression Data Using Real-Time Quantitative PCR and the 2- $\Delta \Delta$ CT Method. Methods 2001; 25: 402-408. https://doi. org/10.1006/meth.2001.1262

[6] GUPTA RA, SHAH N, WANG KC, KIM J, HORLINGS HM et al. Long non-coding RNA HOTAIR reprograms chromatin state to promote cancer metastasis. Nature 2010; 464: 1071-1076. https://doi.org/10.1038/nature08975

[7] GHAFOURI-FARD S, DASHTI S, FARSI M ,TAHERI M. HOX transcript antisense RNA: An oncogenic lncRNA in diverse malignancies. Exp Mol Pathol 2020; 118: 104578104587. https://doi.org/10.1016/j.yexmp.2020.104578

[8] FOCK KM. Review article: the epidemiology and prevention of gastric cancer. Aliment Pharmacol Ther 2014; 40: 250-260. https://doi.org/10.1111/apt.12814

[9] SIEGEL RL, MILLER KD, JEMAL A. Cancer Statistics, 2017. CA Cancer J Clin 2017; 67: 7-30. https://doi.org/10.3322/ caac. 21387

[10] HAJJARI M, BEHMANESH M, SADEGHIZADEH M ,ZEINODDINI M. Up-regulation of HOTAIR long non-coding RNA in human gastric adenocarcinoma tissues. Med Oncol 2013;30:670-673.https://doi.org/10.1007/s12032-013-0670-0

[11] ARITA T, ICHIKAWA D, KONISH H, KOMATSU S, SHIOZAKI A et al. Circulating long non-coding RNAs in plasma of patients with gastric cancer. Anticancer Res 2013; 33: 3185-3193.

[12] ELSAYED ET, SALEM PE, DARWISH AM, FAYED HM. Plasma long non-coding RNA HOTAIR as a potential biomarker for gastric cancer. Int J Biol Markers 2018; 33: 528 533. https://doi.org/10.1177/1724600818760244

[13] LIU XH, SUN M, NIE FQ, GE YB, ZHANG EB et al. Lnc RNA HOTAIR functions as a competing endogenous RNA to regulate HER2 expression by sponging miR-331-3p in gastric cancer. Mol Cancer 2014; 13: 92-105. https://doi. org/10.1186/1476-4598-13-92

[14] LEE NK, LEE JH, PARK CH, YU D, LEE YC et al. Long non-coding RNA HOTAIR promotes carcinogenesis and invasion of gastric adenocarcinoma. Biochem Biophys Res Commun 2014; 451: 171-178. https://doi.org/10.1016/j. bbrc.2014.07.067

[15] JIANG D, LI H, XIANG H, GAO M, YIN C et al. Long Chain Non-Coding RNA (lncRNA) HOTAIR Knockdown Increases miR-454-3p to Suppress Gastric Cancer Growth by Targeting STAT3/Cyclin D1. Med Sci Monit 2019; 25: 1537-1548. https://doi.org/10.12659/MSM.913087

[16] XU ZY, YU QM, DU YA, YANG LT, DONG RZ et al. Knockdown of long non-coding RNA HOTAIR suppresses tumor invasion and reverses epithelial-mesenchymal transition in gastric cancer. Int J Biol Sci 2013; 9: 587-597. https://doi. org/10.7150/ijbs.6339

[17] CHEN WM, CHEN WD, JIANG XM, JIA XF, WANG HM et al. HOX transcript antisense intergenic RNA represses E-cadherin expression by binding to EZH2 in gastric cancer. World J Gastroenterol 2017; 23: 6100-6110. https://doi. org/10.3748/wjg.v23.i33.6100
[18] SONG Y, WANG R, LI LW, LIU X, WANG YF et al. Long non-coding RNA HOTAIR mediates the switching of histone $\mathrm{H} 3$ lysine 27 acetylation to methylation to promote epithelial-to-mesenchymal transition in gastric cancer. Int J Oncol 2019; 54: 77-86. https://doi.org/10.3892/ijo.2018.4625

[19] LIU YW, SUN M, XIA R, ZHANG EB, LIU XH et al. LincHOTAIR epigenetically silences miR34a by binding to PRC2 to promote the epithelial-to-mesenchymal transition in human gastric cancer. Cell Death Dis 2015; 6: e1802-1810. https://doi.org/10.1038/cddis.2015.150

[20] GöKMEN-POLAR Y, VLADISLAV IT, NEELAMRAJU Y, JANGA SC, BADVE S. Prognostic Impact of HOTAIR Expression is Restricted to ER-Negative Breast Cancers. Sci Rep 2015; 5: 8765-8770. https://doi.org/10.1038/srep08765

[21] WEI Z, CHEN L, MENG L, HAN W, HUANG L et al. LncRNA HOTAIR promotes the growth and metastasis of gastric cancer by sponging miR-1277-5p and upregulating COL5A1. Gastric Cancer 2020; 23: 1018-1032. https://doi. org/10.1007/s10120-020-01091-3

[22] ZHANG J, QIU WQ, ZHU H, LIU H, SUN JH et al. HOTAIR contributes to the carcinogenesis of gastric cancer via modulating cellular and exosomal miRNAs level. Cell Death Dis 2020; 11: 780-794. https://doi.org/10.1038/s41419-02002946-4

[23] CHAO P, YONGHENG F, JIN Z, YU Z, SHIYONG Y et al. lncRNA HOTAIR knockdown suppresses gastric cancer cell biological activities. Food Sci Nutr 2021; 9: 123-134. https:// doi.org/10.1002/fsn3.1970

[24] CHENG C, QIN Y, ZHI Q, WANG J, QIN C. Knockdown of long non-coding RNA HOTAIR inhibits cisplatin resistance of gastric cancer cells through inhibiting the PI3K/Akt and Wnt/beta-catenin signaling pathways by up-regulating miR34a. Int J Biol Macromol 2018; 107: 2620-2629. https://doi. org/10.1016/j.ijbiomac.2017.10.154

[25] YAN J, DANG YN, LIU SY, ZHANG YF, ZHANG GX. LncRNA HOTAIR promotes cisplatin resistance in gastric cancer by targeting miR-126 to activate the PI3K/AKT/MRP1 genes. Tumour Biol 2016; 37: 16345-16355. https://doi. org/10.1007/s13277-016-5448-5

[26] XUN J, WANG CF, YAO JN, GAO B, ZHANG LF. Long NonCoding RNA HOTAIR Modulates KLF12 to Regulate Gastric Cancer Progression via PI3K/ATK Signaling Pathway by Sponging miR-618. OncoTargets and Therapy 2019; 12: 10323-10334. https://doi.org/10.2147/OTT.S223957

[27] BIE L, LUO S, LI D, WEI Y, MU Y et al. HOTAIR Competitively Binds MiRNA330 as a Molecular Sponge to Increase the Resistance of Gastric Cancer to Trastuzumab. Curr Cancer Drug Targets 2020; 20: 700-709. https://doi.org/10.2174/ 1568009620666200504114000

[28] WU X, ZHOU J, WU Z, CHEN C, LIU J et al. miR-101-3p Suppresses HOX Transcript Antisense RNA (HOTAIR)-Induced Proliferation and Invasion Through Directly Targeting SRF in Gastric Carcinoma Cells. Oncol Res 2017; 25: 1383 1390. https://doi.org/10.3727/096504017X14879366402279

[29] HAJJARI M, RAHNAMA S. Association Between SNPs of Long Non-coding RNA HOTAIR and Risk of Different Cancers. Front Genet 2019; 10: 113-116. https://doi.org/10.3389/ fgene.2019.00113 
[30] PAN W, LIU L, WEI J, GE Y, ZHANG J et al. A functional lncRNA HOTAIR genetic variant contributes to gastric cancer susceptibility. Molecular Carcinogenesis 2014; 55: 90-96. https://doi.org/10.1002/mc.22261

[31] DU ML, WANG WZ, JIN H, WANG QY, GE YQ et al. The association analysis of lncRNA HOTAIR genetic variants and gastric cancer risk in a Chinese population. Oncotarget 2015; 6: 31255-31262. https://doi.org/10.18632/oncotarget.5158

[32] GUO W, DONG Z, BAI Y, GUO Y, SHEN S et al. Associations between polymorphisms of HOTAIR and risk of gastric cardia adenocarcinoma in a population of north China. Tumour Biol 2014; 36: 2845-2854. https://doi.org/10.1007/ s13277-014-2912-y

[33] BAYRAM S, SÜMBÜL AT, DADAŞ E. A functional HOTAIR rs12826786 C $>\mathrm{T}$ polymorphism is associated with breast cancer susceptibility and poor clinicopathological characteristics in a Turkish population: a hospital-based case-control study. Tumor Biology 2015; 37: 5577-5584. https://doi. org/10.1007/s13277-015-4430-y

[34] ÜLGER Y, DADAŞ E, YALINBAŞ KAYA B, SÜMBÜL AT, GENÇ A et al. The analysis of lncRNA HOTAIR rs12826786 $\mathrm{C}>\mathrm{T}$ polymorphism and gastric cancer susceptibility in a Turkish population: lack of any association in a hospitalbased case-control study. Ir J Med Sci 2017; 186: 859-865. https://doi.org/10.1007/s11845-017-1596-X

[35] BAYRAM S, ULGER Y, SUMBUL AT, KAYA BY, RENCUZOGULLARI A et al. A functional HOTAIR rs920778 polymorphism does not contributes to gastric cancer in a Turkish population: a case-control study. Fam Cancer 2015; 14 : 561-567. https://doi.org/10.1007/s10689-015-9813-0

[36] MINN AKK, SATO N, MIENO MN, ARAI T, MURAMATSU M. Association study of long non-coding RNA HOTAIR rs920778 polymorphism with the risk of cancer in an elderly Japanese population. Gene 2019; 729: 144263-144289. https://doi.org/10.1016/j.gene.2019.144263
[37] LI YF, ZHANG Z, YANG Y, MA J. Long Noncoding RNA HOX Transcript Antisense RNA Gene rs17720428 Single Nucleotide Polymorphism Is Associated with Gastric Cancer Risk and Prognosis. Genet Test Mol Biomarkers 2020; 24: 38-46. https://doi.org/10.1089/gtmb.2019.0140

[38] ABDI E, LATIFI-NAVID S, ZAHRI S, KHOLGHI-OSKOOEI V, MOSTAFAIY B et al. SNP-SNP interactions of oncogenic long non-coding RNAs HOTAIR and HOTTIP on gastric cancer susceptibility. Sci Rep 2020; 10: 16763-16774. https://doi.org/10.1038/s41598-020-73682-0

[39] PETKEVICIUS V, STRELECKIENE G, BALCIUTE K, LINK A, LEJA $\mathrm{M}$ et al. Association of Long Non-Coding RNA Polymorphisms with Gastric Cancer and Atrophic Gastritis. Genes (Basel) 2020; 11: 1505-1515. https://doi.org/10.3390/ genes11121505

[40] LIU FT, ZHOU L, QIU C, XIA GF, ZHU PQ et al. Association of the HOTAIR rs4759314 polymorphism with cancer risk: a meta-analysis. J BUON 2016; 21: 1016-1023.

[41] ZHANG J, LIU X, YOU LH, ZHOU RZ. Significant association between long non-coding RNA HOTAIR polymorphisms and cancer susceptibility: a meta-analysis. Onco Targets Ther 2016; 9: 3335-3343. https://doi.org/10.2147/OTT. S107190

[42] ZHANG ZX, TONG X, ZHANG WN, FU WI. Association between the HOTAIR polymorphisms and cancer risk: an updated meta-analysis. Oncotarget 2017; 8: 4460-4470. https://doi.org/10.18632/oncotarget.13880

[43] QI QC, WANG JW, HUANG B, CHEN AJ, LI G et al. Association of HOTAIR polymorphisms rs4759314 and rs920778 with cancer susceptibility on the basis of ethnicity and cancer type. Oncotarget 2016; 7: 38775-38784. https://doi. org/10.18632/oncotarget.9608

[44] GE Y, JIANG R, ZHANG M, WANG H, ZHANG L et al. Analyzing 37,900 samples shows significant association between HOTAIR polymorphisms and cancer susceptibility: a meta-analysis. Int J Biol Markers 2017; 32: e231-e242. https://doi.org/10.5301/jbm.5000235 